\title{
Cervical cancer risk factors: classification and mining associations
}

\author{
Arif-Ul-Islam*, Shamim H. Ripon, Nuruddin Qaisar Bhuiyan \\ Computer Science and Engineering Department, East West University, Bangladesh, India \\ *Corresponding author, e-mail: a.rifislamm.cse@gmail.com
}

\begin{abstract}
Women of all over the world suffer from a common cancer, named Cervical cancer. Cervical cancer cells grow slowly at the cervix. This cancer can be avoided if it is recognized and handled in its first stage. Now it is a key challenge for Medical experts to identify such cancer before it develops extremely. Nowadays, data mining models are popularly used to extract hidden patterns from huge medical dataset. This paper introduces data mining techinques for classification and finding associations in order to detect Cervical cancer at early stage. After preprocessing, the dataset was tested on Decision Tree, Random Forest, Logistic Model Tree and Artificial Neural Network. These methods achieve considerable success in case of both $K$-fold cross validations and randomly split dataset. Association rules has been established for detecting comparatively riskier factors which are more responsible for cancer development. The proposed methodology can help Medical experts to conduct their research on Cervical cancer.
\end{abstract}

Keywords: association rules, cervical cancer, classification, data mining,

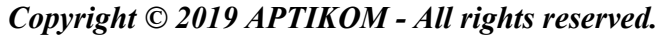

\section{Introduction}

In our body, cells are growing and dividing throughout the life. This is maintained by various genes. Sometimes this process is hampered by different factors such as smoking, taking chemicals or substances as well as certain behaviors. If this happens, the old cells of human body do not die in time and new cells come in improper time. These cells can make tumor [1]. And later, cancer cells spread other tissues. Cervical cancer is one of the common cancers that develops in women. Women of all age groups are the sufferers from this deadly disease. It is deadly because it may not produce any symptoms during the early stage. Symptoms appears when the cervical cancer cells block the tube that carry urine from the bladder and start to spread to other parts close to the cervix like bones, liver, lymph nodes and lungs. In this final stage, the symptoms can be leg pain, fatigue, weight loss, vaginal bleeding, bone fractures and pelvic pain. In 2012, there were 530,000 new cases which was $7.9 \%$ of all female cancers [2]. It is estimated that 4,170 women will die because of cervical cancer in 2018 [3]. Human papillomavirus (HPV), smoking, multiple pregnancies, taking hormonal contraceptives, many sexual partners, early sexual activity, a weak immune system etc. are the risk factors for developing cervical cancer. Among them HPV is the major cause and it can be more harmful if the HPV-infected women smokes [5]. Researcher have developed different types of screening which are applied to identify symptoms in the early stage. Regular screening program for cervical cancer can drop the death rate [4]. But women in developing countries are not aware of this routine screening. Moreover, there are lack of physician expertise and medical equipment which make cervical cancer a deadliest disease [5]. Building a model to detect the cervical cancer in the primary stage based on the risk factors can improve the survival rate. Large amounts of cancer data are available to the medical research community.

Many researches have been conducted in the field of classification of cervical cancer patients and analysis of risk factors of this disease. Authors of [5] introduced Support Vector Machine (SVM) approach for diagnosis of cervical cancer. To diagnose the malignant cancer samples, another two SVM methods, SVM-RFE and SVM-PCA were proposed. As symptoms of cervical cancer appear during the final stage, Sunny Sharma applied Decision Tree for recognizing the stage of this disease [6]. In [7], authors discussed a method to detect and classify the stage of cervical cancer by texture and geometric features of Pap smear images. They used combined approach of Principle Component Analysis (PCA) and multi SVM and achieved accuracy of 95\%. In [8], authors proposed a classification system. They used medical records which had been collected from Pap smear test. To do classification, Naïve Bayes, Support Vector Machines (SVM), and Random Forest Tree were used. Among these techniques, Random 
Forest was proved to be best classifier. They analyzed results by accuracy, precision, recall, and ROC curve. Another classification system of cervical cancer was presented by Muhammed Fahri Ünlerşen, Kadir Sabanci and Muciz Ozcan in [9]. They classified cancer patient using Multilayer Perceptron (MP), BayesNet (BN) and k-Nearest Neighbor (KNN). M. Anousouya Devi et al. [10] discussed different ANN structures for classification of abnormal cervical cancer cells and normal cells. The classification of these cells using ANN was more accurate than normal screening methods [10]. Procedures of detecting infected area of cervical cancer cells were described in [11]. Edge detection and hybrid segmentation work on images to find the tumor.

Our research aim is to build a model by which risk factors of cervical cancer would be analyzed in terms of classification and association rules. To apply detection process, Artificial Neural Network (ANN), Decision Tree (DT), Random Forest (RF) and Logistic Model Tree (LMT) are used. Classification can't be the only way to experiment the risk factors of cancer. To overcome this, next target of this research is to identify associations among the risk factors. Apriori algorithm has been applied to identify associations among the risk factors that are present in the experimented dataset. Support, confidence as well as correlation help to identify only the significant rules.

\section{Proposed Model}

The cervical cancer dataset, which is used in this proposed model, was collected at Hospital Universitariode Caracas in Caracas, Venezuela [12]. It contains medical records of 858 patients. Out of these patients, number of the healthy women is 803 and on the other hand number of patients with cervical cancer diagnosis is 55. This dataset has 32 risk factors, including patient habits, past medical records etc. and four target classes. Target labels or classes are Hinselmann, Schiller, Cytology and Biopsy. Some patients do not answer all the questions for individual privacy reason. The proposed method is divided into three parts- 1) preprocessing 2) classification and 3) generating the association rules. Figure 1 shows the structure of the model. In this model there are three phases.

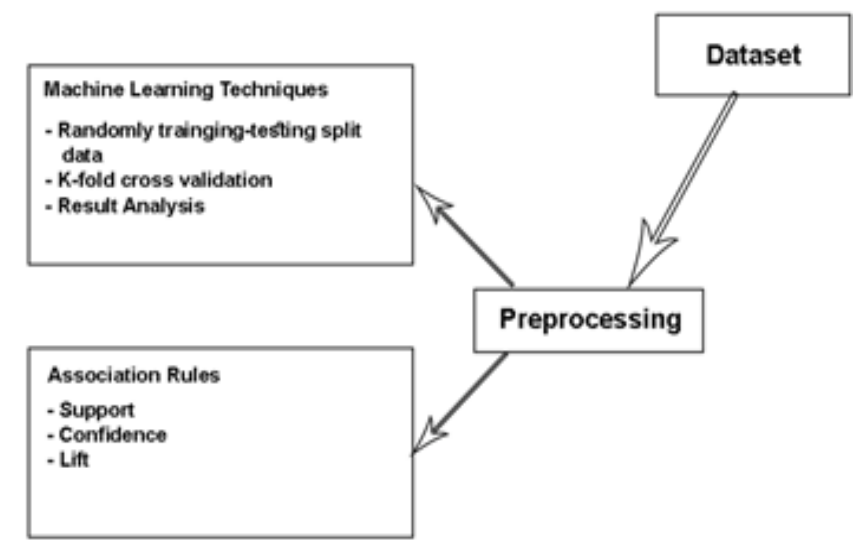

Figure 1. Proposed model structure

\subsection{Preprocessing}

Cervical cancer dataset need to be preprocessed to discover hidden information. So, in first phrase, we preprocess the dataset by handling missing values and imbalanced class levels.

\subsection{Classification}

In the second step, prediction of how many patients are affected by cancer is found by imposing the dataset into the data mining techniques. Data mining techniques are-
a. Artificial Neural Network (ANN)
b. Decision Tree (DT)
c. Random Forest (RF) and
d. Logistic Model Tree (LMT) 
At first, classification process is applied on randomly split dataset. Later, to detect the model does overfit or not, K-fold validation method is applied. Results are analyzed by accuracy, kappa, precision and recall.

\subsection{Associations}

Association process helps to find the strongly connected attributes in the dataset. In our last phase, associations among the attributes and class levels is determined for recognizing which attributes are positively connected with the cancer.

\section{Research Method}

This section describes the implementation of the proposed method.

\subsection{Preprocessing}

Cervical cancer dataset has 32 feature and 4 class levels. It contains many missing values. 27 and 28 number attributes (STDs: Time since first diagnosis and STDs: Time since last diagnosis) have more missing data than other attirbutes. These two columns will have no better impact on the classification and associations. We drop these two attributes. After dropping, there are 30 attributes. Some other columns also have few missing cells. Among those columns, some are categorical and some are discrete values. To overcome this curse, missing values of categorical columns and discrete columns are replaced by the mode and the mean values of that columns respectively. Class levels are imbalanced. Number of instances belongs to "no" class is very high than the number of instances which belongs to "yes" class. Because of this situation, result might be inaccurate. So, for getting the accurate result, we balance our dataset using resample filter of WEKA tool. Table 1 descrives numerical description of attributes of the dataset.

Table 1. Attributes description after preprocessing

\begin{tabular}{llllll}
\hline Attributes & Mean & S. Dev. & Attributes & Mean & S. Dev. \\
\hline Age & 26.8205 & 8.497948 & STDs: vaginalcondylomatosis & 0.004662 & 0.068159 \\
\# of partners & 2.51165 & 1.644759 & STDs: vulvo-perinealcondylomatosis & 0.050117 & 0.218313 \\
Age of 1st intercourse & 16.9790 & 2.797653 & STDs: syphilis & 0.020979 & 0.143398 \\
\# of pregnancies & 2.19230 & 1.434395 & STDs: pelvic inflammatory disease & 0.001166 & 0.034139 \\
Smokes & 0.14335 & 0.350641 & STDs: genital herpes & 0.001166 & 0.034139 \\
Smokes years & 1.20124 & 4.060623 & STDs: molluscumcontagiosum & 0.001166 & 0.034139 \\
Smokes packs/year & 0.44627 & 2.210351 & STDs: AIDS & 0 & 0 \\
Hormonal contraceptives & 0.68648 & 0.464194 & STDs: HIV & 0.020979 & 0.143398 \\
Hormonal contraceptives years & 1.97239 & 3.597888 & STDs: Hepatitis B & 0.001166 & 0.034139 \\
IUD & 0.09673 & 0.295771 & STDs: HPV & 0.002331 & 0.048252 \\
IUD years & 0.44460 & 1.814218 & STDs: Number of diagnosis & 0.087413 & 0.302545 \\
STDs & 0.09207 & 0.2893 & Dx: Cancer & 0.020979 & 0.143398 \\
STDs number & 0.15501 & 0.529617 & Dx: CIN & 0.01049 & 0.101939 \\
STDs: condylomatosis & 0.05128 & 0.220701 & Dx: HPV & 0.020979 & 0.143398 \\
STDs: cervicalcondylo- mitosis & 0 & 0 & DX & 0.027972 & 0.164989 \\
\hline
\end{tabular}

\subsection{Classification:}

ANN and tree models were perfectly implemented for healthcare system [13]. Because of this successful implementation, we used ANN, Decision Tree, Random Forest in our model for classification purpose.

\subsubsection{ANN}

Concepts of Artificial Neural Network comes from the idea of working of human brains. The network is collection of three different layers: input layer, hidden layer and output layer. Each layer consists of multiple single processing units named perceptron. These units perform complex computations parallelly. The signals given to the neural network are propagated through the layer by layer. Units of the input layer takes input feature vectors and this layer is connected to the hidden layer. The output layer provides predictions of the class levels.

Perceptron does computations by three steps.

a. All the inputs $(\mathrm{i} 1, \mathrm{i} 2, \ldots$, in) are multiplied by the weights $(w 1, w 2, \ldots, w n)$.

APTIKOM J. CSIT Vol. 4, No. 1, 2019: 8-18 
b. Then all the multiplied values are added.

c. Later, the weighted sum is imposed to the activation function.

Table 2 presents the information about parameters that are were in the implemented ANN.

Table 2. Details properties of implemented ANN in proposed method

\begin{tabular}{ll}
\hline Parameters & Values \\
\hline Type & Backpropagation \\
Hidden Layers & 4 \\
Learning Rate & 0.3 \\
\hline
\end{tabular}

\subsubsection{Decision Tree}

Decision tree is a supervised algorithm. This creates a training model based on decision rules. This model can predict class labels by building a tree structure. It has one root, multiple internal nodes and leaf nodes. Internal nodes and leaf nodes correspond to attributes and class levels respectively. After building the tree structure, when a new instance comes, it is matched with extracted rules from the decision tree. We use J48 decision tree. Details of implemented Decision Tree model are shown in Table 3.

Table 3. Details properties of implemented Decision Tree in proposed method

\begin{tabular}{ll}
\hline Parameters & Values \\
\hline Minimum number of instances per leaf & 2 \\
Confidence Factor & 0.25 \\
\hline
\end{tabular}

\subsubsection{Random Forest}

Random forest is ensemble of decision tree. Simply, random forest creates multiple decision trees and merge them to find the overall best results. It is a supervised algorithm like decision tree. At a time, it can do classification and regression. When splitting a node, it generally searches for the best attribute among random features. This process makes the model better. Threshold can be used to make the model more random. Details properties of implemented Random Forest in proposed method as shown in Table 4.

Table 4. Details properties of implemented Random Forest in proposed method

\begin{tabular}{ll}
\hline Parameters & Values \\
\hline Maximum depth of the tree & 0 [Unlimited] \\
Number of iterations & 100 \\
\hline
\end{tabular}

\subsection{Association Rules}

Apriori is a popular data mining technique. It is used to find correlations, patterns from the dataset. A huge dataset can provide many rules including unwanted associations. Different types of constraints such as Support, Confidence, Lift, Conviction are applied for extracting only important rules. Support indicates of the frequency of items in the dataset. Confidence represents the number of times the if/then rules become true. Lift is used to find the positively correlated rules. Details properties of implemented Apriori Algorithm in proposed method as shown in Table 5.

Table 5. Details properties of implemented Apriori Algorithm in proposed method

\begin{tabular}{ll}
\hline Parameters & Values \\
\hline Minimum support & 0.6 \\
Minimum Confidence & $80 \%$ \\
\hline
\end{tabular}

\section{Results and Analysis}

This section deals with the analysis of results. The proposed methodology was implemented on a system having 8GB RAM and 3.5 GHz Intel Core i-7 processor using WEKA 3.8 tool. During the 
preprocessing, cervical cancer dataset was divided into 4 individual datasets according to 4 classes. So, each dataset had one class attribute: Hinselmann, Schiller, Citology and Biopsy. On cervical cancer preprocessed datasets, two types of analysis had been performed. In the first part, classification of cancer patients had been done by 4 different data mining techniques. In the second study, association rules among the attributes had been generated by apriori algorithm. K-fold cross validation technique widely works to analyze the performance of data mining models [14]. In this approach, $\mathrm{k}$ is the number of parts of the dataset. k-1 folds and kth fold are used as the training set and testing set respectively. This method is very much helpful to avoid overfitting problem.

\subsection{Classification Performance}

To evaluate the results of algorithms, here five methods were used which are accuracy, precision, recall, f1-score and kappa. These parameters are used to judge the techniques based on actual result and predicted outcome. Actual result is true level of the instance and predicted outcome is what model predicts the class level of that instance. High values of these mean a good prediction of the data mining models.

Accuracy: It is one of the simplest performance measures and simply the ratio of correctly predicted observation and total observation. Accuracy is calculated as the summation of true positive and true negative divided by total number of subject in the study.

$$
\text { Total Accuracy }=(T P+T N) /(T P+T N+F P+F N)
$$

Precision: Refers to the ability of a classification model to identify only the relevant points. Precision is calculated as the number of true positive divided by the summation of the number of true positive and true negative.

$$
\text { Precision }=T P /(T P+T N)
$$

Recall: It refers to the ability of a model to find all the relevant cases within a dataset. Recall is calculated as the number of true positive divided by the summation of the number of true positive and false negative.

$$
\text { Recall }=T P /(T P+F N)
$$

Kappa: Kappa is an important measure of classifier performance on imbalanced dataset. It compares an observed accuracy with an expected accuracy (random chance). Kappa is calculated as total accuracy minus random accuracy divided by one minus random accuracy.

$$
\begin{aligned}
& \text { Kappa }=(\text { Total Accuracy }- \text { Random Accuracy }) /(1-\text { Ramdom Accuracy }) \\
& \text { Total Accuracy }=(T P+T N) /(T P+T N+F P+F N) \\
& \text { Random Accuracy }=\frac{(T N+F P) *(T N+F N)+(F N+T P) *(F P+T P)}{\text { Total } * \text { Total }} \\
& \text { Total }=T P+T N+F P+F N
\end{aligned}
$$

Value of kappa parameter is between 0 and 1(inclusive).

F1-score: Refers to the weighted average of precision and recall. It takes precision and recall and gives an unbiased accuracy measure.

$$
\text { F1score }=(2 * \text { precision } * \text { recall }) /(\text { precision }+ \text { recall })
$$

\subsubsection{Analysis of model performance on randomly split training-testing dataset}

Interpretation: On Biopsy dataset, DT, RF and LMT each claims accuracy over $99 \%$ and precision of 99.6. The best recall giving classifier is RF and the lowest accuracy reporting classifier is ANN. RF claims the best kappa and f1-score value among the classifiers which is 97 and 99.8 respectively. For this dataset, RF performs better than any other classifier. Performance comparison of four classifiers on Biopsy dataset shown in Figure 2. Comparison of Accuracy, Recall, Precision, Kappa, F1-score of different algorithms on Biopsy dataset as shown in Table 6. 
Table 6. Comparison of Accuracy, Recall, Precision, Kappa, F1-score of different algorithms on Biopsy dataset

\begin{tabular}{llllll}
\hline & Accuracy (100) & Precision (100) & Recall (100) & Kappa (1) & F1-score (100) \\
\hline ANN & 96.23 & 96 & 100 & 0.7 & 98 \\
DT & 99.31 & 99.6 & 99.6 & 0.96 & 99.6 \\
RF & 99.65 & 99.6 & 100 & 0.97 & 99.8 \\
LMT & 99.31 & 99.6 & 99.6 & 0.95 & 99.6 \\
\hline
\end{tabular}

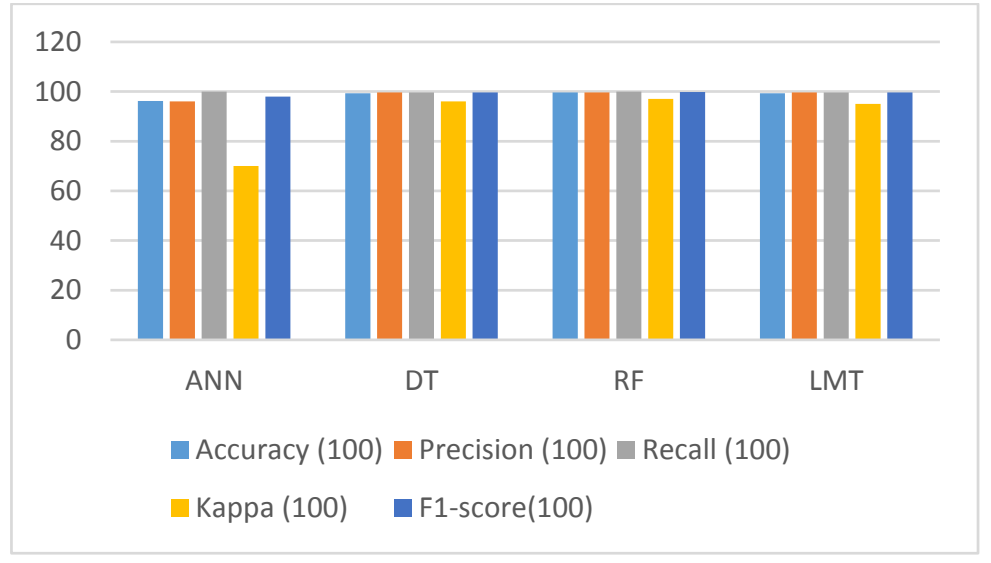

Figure 2. Performance comparison of four classifiers on Biopsy dataset.

Interpretation: On Citology dataset DT reports better accuracy than others which is 97.94, best f1-score of 98.9 and claims the best precision of 97.8. All of the classifiers give same recall value of 100 and LMT gives the best kappa value. Performance comparison of four classifiers on Citology dataset shown in Figure 3. Comparison of Accuracy, Recall, Precision, Kappa and F1-score of different algorithms on Citology dataset as shown in Table 7.

Table 7. Comparison of Accuracy, Recall, Precision, Kappa and F1-score of different algorithms on

\begin{tabular}{llllll}
\multicolumn{7}{c}{ Citology dataset } \\
\hline & Accuracy (100) & Precision (100) & Recall (100) & Kappa (1) & F1-score (100) \\
\hline ANN & 96.57 & 96.4 & 100 & 0.71 & 98.2 \\
DT & 97.94 & 97.8 & 100 & 0.83 & 98.9 \\
RF & 96.57 & 96.4 & 100 & 0.71 & 98.2 \\
LMT & 96.57 & 96.4 & 100 & 0.95 & 98.2 \\
\hline
\end{tabular}

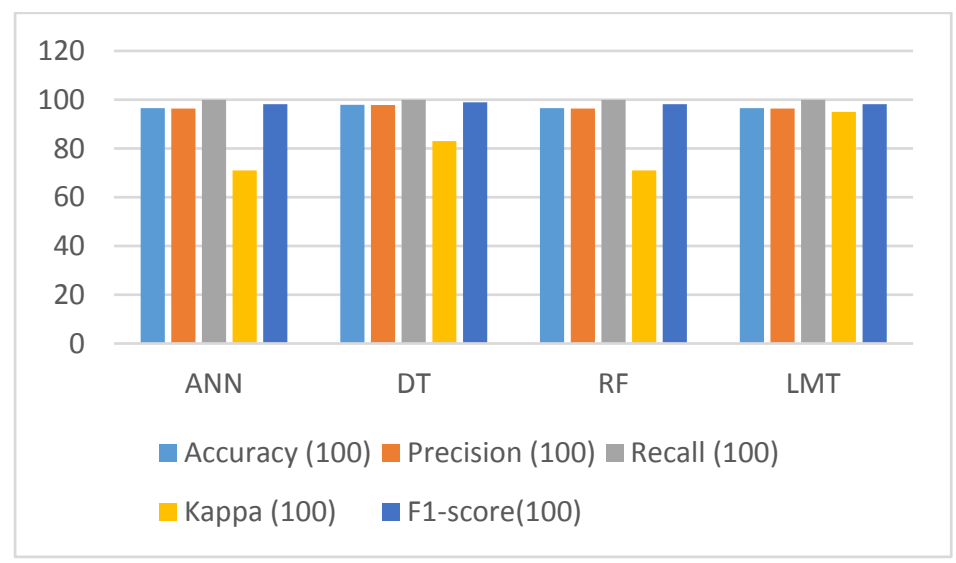

Figure 3. Performance comparison of four classifiers on Citology dataset. 
Interpretation: On Hinselmann dataset RF gives the highest value for all the comparison factors. Hence, RF performs better than any other classifiers used on this dataset. Performance comparison of four classifiers on Hinselmann dataset shown in Figure 4. Comparison of Accuracy, Recall, Precision, Kappa and F1-score of different algorithms on Hinselmann dataset as shown in Table 8.

Table 8. Comparison of Accuracy, Recall, Precision, Kappa and F1-score of different algorithms on Hinselmann dataset

\begin{tabular}{cccccc}
\hline & Accuracy (100) & Precision (100) & Recall (100) & Kappa (1) & F1-score (100) \\
\hline ANN & 98.63 & 98.6 & 100 & 0.85 & 99.3 \\
DT & 98.97 & 98.9 & 100 & 0.89 & 99.5 \\
RF & 100 & 100 & 100 & 1 & 100 \\
LMT & 99.65 & 99.6 & 100 & 0.96 & 99.8 \\
\hline
\end{tabular}

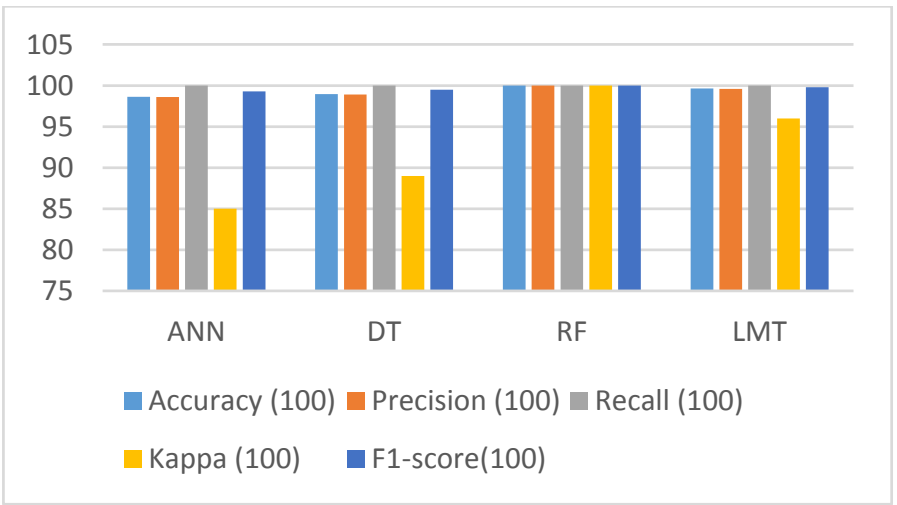

Figure 4. Performance comparison of four classifiers on Hinselmann dataset.

Interpretation: On Schiller dataset, though all the classifiers claim best recall value of 100 but LMT gives the highest value for all other factors. Therefore, LMT performs better than any other classifiers used on this dataset. Performance comparison of four classifiers on Schiller dataset shown in Figure 5. Comparison of Accuracy, Recall, Precision, Kappa and F1-score of different algorithms on Schiller dataset as shown in Table 9

Table 9. Comparison of Accuracy, Recall, Precision, Kappa and F1-score of different algorithms on Schiller dataset

\begin{tabular}{llllll}
\hline & Accuracy (100) & Precision (100) & Recall (100) & Kappa (1) & F1-score (100) \\
\hline ANN & 96.23 & 95.9 & 100 & 0.77 & 97.9 \\
DT & 95.8 & 95.6 & 100 & 0.74 & 97.7 \\
RF & 97.6 & 97.4 & 100 & 0.86 & 98.7 \\
LMT & 97.94 & 97.7 & 100 & 0.88 & 98.9 \\
\hline
\end{tabular}




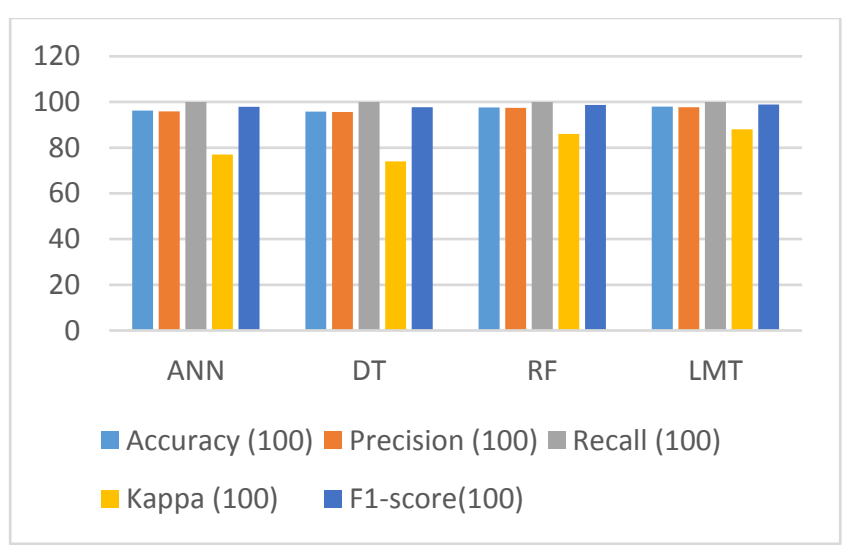

Figure 5. Performance comparison of four classifiers on Schiller dataset.

\subsubsection{Analysis of model performance on 10-fold cross validation}

Interpretation: RF showed better performance over other models. Interpretation: Both RF and LMT having maximum possible value of each factors perform better than other two classifiers. Performance comparison of four classifiers on Biopsy dataset shown in Figure 6. Performance comparison of four classifiers on Citology dataset shown in Figure 7. Comparison of Accuracy, Recall, Precision, Kappa of different algorithms on Biopsy dataset as shown in Table 10. Comparison of Accuracy, Recall, Precision, Kappa of different algorithms on Citology dataset as shown in Table 11.

Table 10. Comparison of Accuracy, Recall, Precision, Kappa of different algorithms on Biopsy dataset

\begin{tabular}{llllll}
\hline & Accuracy (100) & Precision (100) & Recall (100) & Kappa (1) & F1-score (100) \\
\hline ANN & 98.60 & 98.9 & 99.6 & 0.87 & 99.3 \\
DT & 99.30 & 99.9 & 99.4 & 0.94 & 99.6 \\
RF & 99.76 & 99.8 & 100 & 0.98 & 99.9 \\
LMT & 99.53 & 99.9 & 99.6 & 0.96 & 99.8 \\
\hline
\end{tabular}

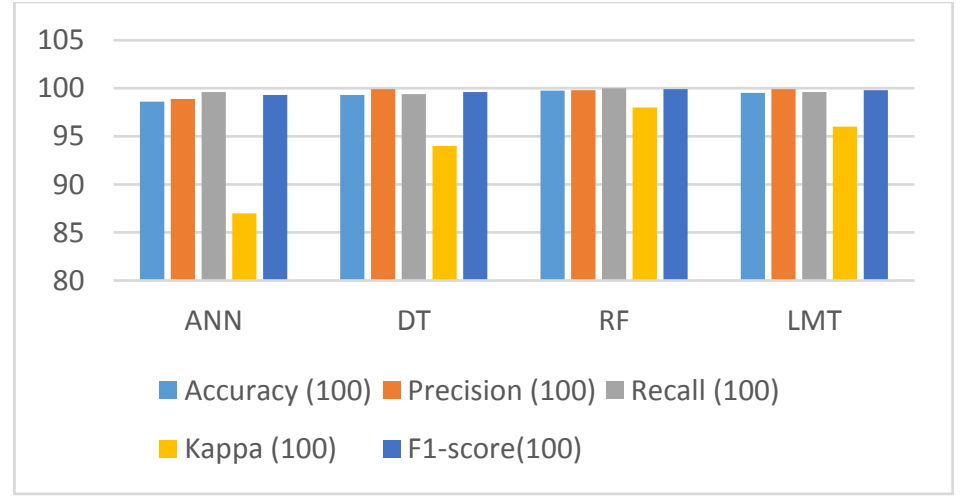

Figure 6. Performance comparison of four classifiers on Biopsy dataset.

Table 11. Comparison of Accuracy, Recall, Precision, Kappa of different algorithms on Citology dataset

\begin{tabular}{llllll} 
& Accuracy (100) & Precision (100) & Recall (100) & Kappa (1) & F1-score (100) \\
\hline ANN & 98.01 & 97.8 & 100 & 0.73 & 98.9 \\
DT & 98.01 & 98.0 & 100 & 0.75 & 99 \\
RF & 100 & 100 & 100 & 1 & 100 \\
LMT & 100 & 100 & 100 & 1 & 100 \\
\hline
\end{tabular}




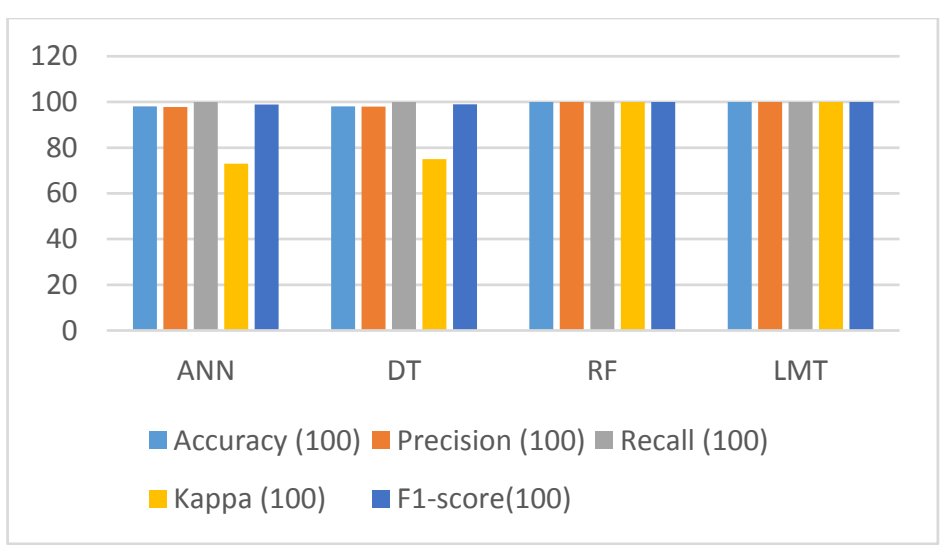

Figure 7. Performance comparison of four classifiers on Citology dataset

Interpretation: DT, RF, LMT all these three classifiers give the same value of comparison matrices which are better than ANN. Performance comparison of four classifiers on Hinselmann dataset shown in Figure 8. Comparison of Accuracy, Recall, Precision, Kappa of different algorithms on Hinselmann dataset as shown in Table 12.

Table 12. Comparison of Accuracy, Recall, Precision, Kappa of different algorithms on

\begin{tabular}{llllll}
\multicolumn{6}{c}{ Hinselmann dataset. } \\
\hline & Accuracy (100) & Precision (100) & Recall (100) & Kappa (1) & F1-score (100) \\
\hline ANN & 99.18 & 99.2 & 100 & 0.88 & 99.6 \\
DT & 99.53 & 99.5 & 100 & 0.937 & 99.8 \\
RF & 99.53 & 99.5 & 100 & 0.937 & 99.8 \\
LMT & 99.53 & 99.5 & 100 & 0.937 & 99.8 \\
\hline
\end{tabular}

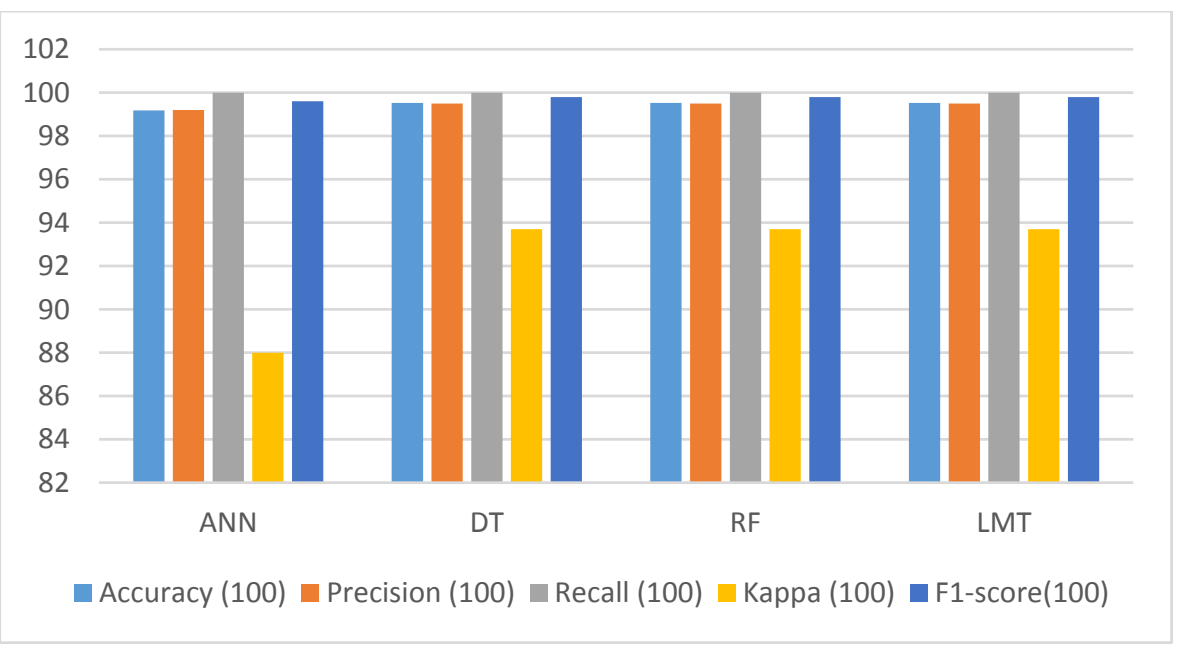

Figure 8. Performance comparison of four classifiers on Hinselmann dataset.

Interpretation: The RF performs best having maximum score in all the comparison parameters. Performance comparison of four classifiers on Schiller dataset shown in Figure 9. Comparison of Accuracy, Recall, Precision, Kappa of different algorithms on Schiller dataset as shown in Table 13. 
Table 13. Comparison of Accuracy, Recall, Precision, Kappa of different algorithms on Schiller dataset.

\begin{tabular}{llllll}
\hline & Accuracy (100) & Precision (100) & Recall (100) & Kappa (1) & F1-score (100) \\
\hline ANN & 98.25 & 98.1 & 100 & 0.87 & 99.1 \\
DT & 98.37 & 98.2 & 100 & 0.88 & 99.1 \\
RF & 99.53 & 99.5 & 100 & 0.96 & 99.7 \\
LMT & 99.41 & 99.5 & 99.99 & 0.96 & 99.7 \\
\hline
\end{tabular}

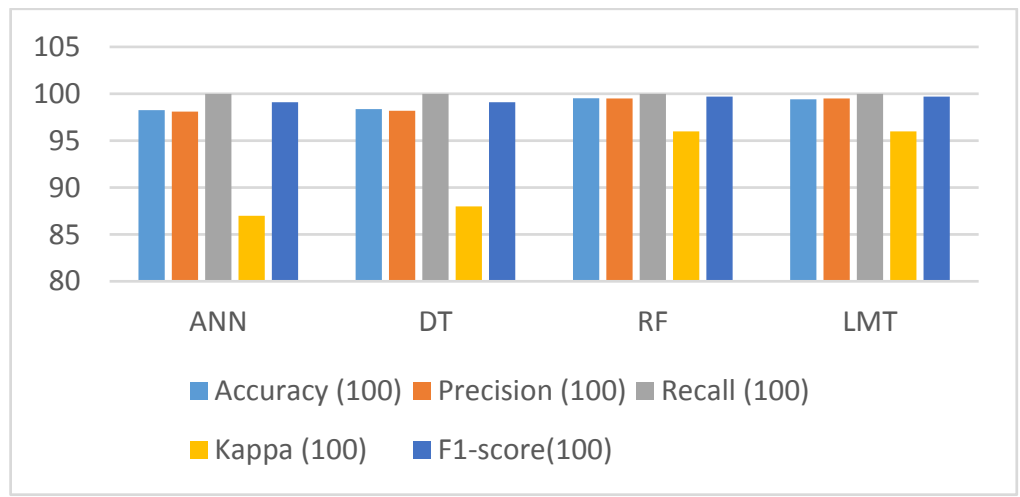

Figure 9. Performance comparison of four classifiers on Schiller dataset.

\subsection{Association Rules Analysis:}

So many rules have been established from implementation of apriori algorithm on four (4) dataset. As research aim is to extract the rules that contain class level "yes", the rules which consist of class level "no" and the rules which only contain attributes (no class levels) are dropped. Then the below rules remain-

a. $\quad$ Age $=20$ \& Hormonal Contraceptives=1->Biopsy

b. Number of Sexual Partners (>=2)->Cytology

c. Hormonal Contraceptives $=1 \&$ Smoke $=1->$ Hinselmann

d. Num of pregnancies $=3$ and Hormonal Contraceptives $=1->$ Schiller

e. First sexual intercourse $=17$ and Hormonal Contraceptives $=1$-> Schiller

The attributes in the rules are identified as the riskiest factors in case of cervical cancer.

Interpretation of the rules:

a. Women of age 20 who take Hormonal Contraceptives,

b. Women who have more than 2 sexual partners,

c. Women who smoke and take Hormonal Contraceptives,

d. Women who have become pregnant 3 times and used to take Hormonal Contraceptives

e. Women whose first sexual intercourse was at age 17 and if they take Hormonal Contraceptives are at risk of cervical cancer.

\section{Conclusion}

In this paper, classification and associations of cervical cancer risk factors are performed. Several approaches are performed for avoiding missing values. Later, based on the risk factors, data mining techniques- DT, RF, LMT and ANN are trained for classification of cervical cancer patients from healthy patients. Their performances are discussed by recall, precision, f1-score and kappa parameters. These models provide reasonable accuracy and these accuracies are close to each other. On average, RF proved to be better than others. Associations among class level 'yes' and the attributes, are inducted using Apriori algorithm. These rules contain the most important risk factors that are truly considered by medical science as dangerous factors for building cervical cancer. This proposed model can be used for recognition of cervical cancer before it develops and necessary precautions can be taken to avoid this. 


\section{References}

[1] Mandal Sudip, Banerjee Indrojit. Cancer Classification Using Neural Network. International Journal of Emerging Engineering Research and Technology. 2015; 3: 172-178.

[2] Cervical cancer, World Health Organization, 2018. [Online]. Available: http://www.who.int/cancer/prevention/diagnosis-screening/cervical-cancer/en/.

[3] Cervical Cancer: Statistics |Cancer.Net, Cancer.Net, 201. [Online]. Available: https://www.cancer.net/cancertypes/cervical-cancer/statistics.

[4] PK Malli, S Nandyal. Machine learning Technique for detection of Cervical Cancer using k-NN and Artificial Neural Network, 2017.

[5] W Wu, H Zhou. Data-Driven Diagnosis of Cervical Cancer With Support Vector Machine-Based Approache. in IEEE Access. 2017; 5: 25189-25195.

[6] S Sharma. Cervical Cancer stage prediction using Decision Tree approach of Machine Learning. International Journal of Advanced Research in Computer and Communication Engineering 2016; 5(4): 345-348.

[7] Debashree Kashyap, Abhishek Somani, Jatin Shekhar, Anupama Bhan, Malay Kishore Dutta, Malay Kishore Dutta, Kamil Riha. Cervical cancer detection and classification using Independent Level sets and multi SVMs. 2016 39th International Conference on Telecommunications and Signal Processing (TSP), Vienna. 2016: pp. 523-528. doi: 10.1109/TSP.2016.7760935

[8] YE Kurniawati, AE Permanasari, S Fauziati. Comparative study on data mining classification methods for cervical cancer prediction using pap smear results, 2016 1st International Conference on Biomedical Engineering (IBIOMED), Yogyakarta, 2016: 1-5. doi: 10.1109/IBIOMED.2016.7869827

[9] Ünlerșen, Muhammed, Sabanci, Kadir, Ozcan, Muciz. Determining Cervical Cancer Possibility by Using Machine Learning Methods. International Journal of Latest Research in Engineering and Technology (IJLRET). 2017; 3: 65-71.

[10] M Anousouya Devi, S Ravi, J Vaishnavi, S Punitha. Classification of Cervical Cancer Using Artificial Neural Networks. Procedia Computer Science. 2016; 89: 465-472. ISSN 1877-0509. https://doi.org/10.1016/j.procs.2016.06.105.

[11] S Garg, S Urooj, R Vijay. Detection of cervical cancer by using thresholding \& watershed segmentation. 2015 2nd International Conference on Computing for Sustainable Global Development (INDIACom). 2015: 555-559.

[12] K Fernandes, JS Cardoso, J Fernandes. Transfer learning with partial observability applied to cervical cancer screening. in Proc. Iberian Conf. Pattern Recognit. Image Anal. 2017: 243-250.

[13] P Ghavami, K Kapur. Prognostics \& artificial neural network applications in patient healthcare. 2011 IEEE Conference on Prognostics and Health Management. QC. 2011: 1-7. doi: 10.1109/ICPHM.2011.6024340

[14] NN Alotaibi, S Sasi. Tree-based ensemble models for predicting the ICU transfer of stroke in-patients, 2016 International Conference on Data Science and Engineering (ICDSE), Cochin, 2016: 1-6. doi: 10.1109/ICDSE.2016.7823951

APTIKOM J. CSIT Vol. 4, No. 1, 2019: 8-18 